\title{
P01-1-4
}

\section{Spatial representation of hippocampal cells in response to aversive stimulus in a T-maze task}

\author{
Sakura Okada ${ }^{1}$, Hideyoshi Igata ${ }^{1}$, Takuya Sasaki $^{1,2}$, Yuji Ikegaya $^{1}$ \\ ${ }^{1}$ Lab. Chem. Pharmacol., Grad. Sch. Pharmaceut. Sci., Univ. Tokyo, Tokyo, Japan, ${ }^{2}$ Precursory Research for \\ Embryonic Science and Technology, Japan Science and Technology Agency, Japan
}

Hippocampal place cells are cells that specifically discharge when an animal enters a specific zone, so-called a place field. In addition, firing patterns of hippocampal cells are subject to non-spatial information, such as colors, objects, or task demands, which is assumed to be a mechanism by which the hippocampus encodes information of individual experiences onto a neuronal spatial framework. Especially, previous studies have shown that place fields shift to a newly rewarded location, suggesting that the distribution of space represented by place cells is not homogeneous in an environment but specific locations related to survival are more strongly represented. However, how alterations in the firing patterns of place cells in response to aversive events encode the locations associated with these aversive events is unknown. Here, we analyzed spiking patterns of place cell ensembles in the dorsal hippocampal CA1 region of rats performing a T-maze alternation task with an aversive air-puff stimulation applied at a specific location on one side of a trajectory. To obtain reward, rats have to overcome the air-puff. The intensity of the air-puff was adjusted so that the rats decreased their running speed before passing the aversive location. The addition of the aversive stimulus induced reorganization of place cell ensembles on both left and right trajectories with and without the aversive stimulus, respectively. Specifically, the animals showed a more abundant spatial representation in the vicinity of the aversive location. Removing the aversive stimulus induced new spatial firing patterns on both of the trajectories that differed from those both before and during application of the aversive stimulus. These results demonstrate that hippocampal spatial maps are flexibly reorganized to represent particular aversive events. 